\title{
Numerical modelling of sawtooth crash using two-fluid equations
}

\author{
Q. Yu, S. Günter and K. Lackner \\ Max-Planck/Princeton Center for Plasma Physics \\ Max-Planck-Institut für Plasmaphysik, 85748 Garching, Germany
}

The nonlinear growth of $m / n=1 / 1$ internal kink modes is studied numerically using twofluid equations. As already reported earlier [S. Günter, et al, Plasma Phys. Control. Fusion, 2015, 57, 014017], short sawtooth crash times $(<100 \mu s)$ are found for typical ASDEX Upgrade parameters, in agreement with experimental observations. These fast sawtooth crashes are associated with large parallel electric field perturbations, giving rise to the generation of suprathermal electrons. Slow sawtooth crashes $(\sim m s)$ are obtained only for a sufficiently small $\left(1-q_{0}\right)$ and a large local electron diamagnetic drift frequency at the $q=1$ surface, where $q_{0}$ is the value of the safety factor $q$ at the magnetic axis. Kink modes are shown to drive sheared plasma rotation, propagating from the $q=1$ surface towards the magnetic axis during the nonlinear phase. After the sawtooth crash, the driven plasma rotation is in the co- (counter-) current direction inside (outside) the $\mathrm{q}=1$ surface, as observed in experiments. 


\section{Introduction}

Sawteeth are one of the most typical instabilities in tokamak plasmas [1], as the plasma current density profiles usually result in values of the safety factor q below one on the magnetic axis. Sawteeth flatten the plasma pressure profiles inside the $q=1$ surface on a very short time scale due to a fast growing $m / n=1 / 1$ magnetic island, caused by an internal kink mode [1-26], where $m$ and $n$ are the poloidal and toroidal mode numbers, respectively. Large amplitude sawtooth crashes can influence the plasma confinement outside the $q=1$ surface by triggering other instabilities such as neoclassical tearing modes [27,28]. For a fusion reactor the $\alpha$-particle confinement in the core region could also be significantly affected by sawteeth. The understanding of the physics processes during sawtooth crashes thus remains to be an important issue.

Since their first discovery [1], sawtooth instabilities have attracted much research interest [2-26]. Using a Sweet-Parker type analysis [29,30], the sawtooth crash time was found by Kadomtsev to be about $\left(\tau_{A} \tau_{R}\right)^{1 / 2}$, where $\tau_{A}$ is the Alfvén time, and $\tau_{R}$ is the resistive time [2]. The ideal $m / n=1 / 1$ mode can also be unstable, but it was shown to saturate at a small amplitude [26]. The nonlinear mode growth is therefore determined by magnetic reconnection. Experimental measurements in high temperature tokamak plasmas showed sawtooth crash times of about $100 \mu \mathrm{s}$ or shorter [3-5], much less than that predicted by Kadomtsev. Wesson suggested that the reconnection would become much faster for high temperature plasmas when taking into account the electron inertia effect [6]. Further studies have also been carried out to include other effects such as anomalous current diffusion [7-10], finite Larmor radius [12, 1416] and the parallel electron viscosity [17] to understand the fast growth of the $m / n=1 / 1$ island. Alternatively, the nonlinear evolution of the internal kink mode has been treated as a sequence of equilibria [11-14]. 
In this paper numerical modelling results of sawtooth crashes, based on two-fluid equations, are presented. As already shown in [31], fast sawtooth crashes, with crash times being about $100 \mu \mathrm{s}$, are found for typical ASDEX Upgrade parameters, in agreement with experimental observations. For such fast reconnection events, strong parallel electric fields are induced in the x-point region of the magnetic island, giving rise to the generation of superthermal electrons. Furthermore, sheared plasma flows are found to be driven by the internal kink mode.

In Section 2 our theoretical model is described. The numerical results obtained with ASDEX Upgrade relevant parameters as input are presented in Section 3. Finally, the discussion and summary are presented in Section 4.

\section{Theoretical model}

The large aspect-ratio tokamak approximation is utilized. The magnetic field is defined as $\boldsymbol{B}=B_{0 t}\left(\boldsymbol{e}_{t}-\boldsymbol{e}_{\theta} k_{t} / k_{\theta}\right)+\nabla \psi \times \boldsymbol{e}_{t}$, where $\psi$ is the helical flux function, $k_{\theta}=m / r$ and $k_{t}=n / R$ are the wave vector in $\boldsymbol{e}_{\theta}$ (poloidal) and $\boldsymbol{e}_{t}$ (toroidal) direction, $r$ and $R$ are the minor and the major radius, and the subscript 0 denotes an equilibrium quantity. The ion velocity is given by $v=v_{\|}$ $\boldsymbol{e}_{\|}+\boldsymbol{v}_{\perp}$, where $v_{\|}$and $\boldsymbol{v}_{\perp}=\nabla \phi \times \boldsymbol{e}_{t}$ are the parallel (to the magnetic field) and the perpendicular velocity, respectively, and $\phi$ is the stream function.

The electron continuity equation, generalized Ohm's law, the equation of motion in the parallel and the perpendicular direction (after taking the operator $\boldsymbol{e}_{t} \cdot \nabla x$ ), and the electron energy transport equation, are solved, to obtain $\psi, v_{\|}, v_{\perp}$, the electron density $n_{e}$ and temperature $T_{e}$. Normalizing the length to the minor radius $a$, the time $t$ to $\tau_{R}, \psi$ to $a B_{0 t}, v$ to $a / \tau_{R}$, and $T_{e}$ and $n_{e}$ to their values at the magnetic axis, where $a$ is the minor radius, and $\tau_{R}=a^{2} / \eta$ is the resistive time, these equations become [32-34] 


$$
\begin{aligned}
& \frac{d n_{e}}{d t}=d_{1} \nabla_{\|} j-\nabla_{\|}\left(n_{e} V_{\|}\right)+\nabla \cdot\left(D_{\perp} \nabla_{e}\right)+S_{n}, \\
& \frac{d \psi}{d t}=E_{0}-\eta j-\frac{\eta}{v_{e i}} \frac{d j}{d t}+\eta \frac{\mu_{e}}{v_{e i}} \nabla_{\perp}^{2} j+\Omega\left(\nabla_{\|} n_{e}+\nabla_{\|} T_{e}\right), \\
& \frac{d V_{\| \mid}}{d t}=-C_{s}^{2} \nabla_{\|} p / n_{e}+\mu \nabla_{\perp}^{2} V_{\|}, \\
& \frac{d U}{d t}=S^{2} \nabla_{\|} j+\mu \nabla_{\perp}^{2} U, \\
& \frac{3}{2} n_{e} \frac{d T_{e}}{d t}=d_{1} T_{e} \nabla_{\|} j-T_{e} n_{e} \nabla_{\|} V_{\|}+n_{e} \nabla \cdot\left(\chi_{\|} \nabla_{\|} T_{e}\right)+n_{e} \nabla \cdot\left(\chi_{\perp} \nabla_{\perp} T_{e}\right)+S_{p},
\end{aligned}
$$

where

$$
\begin{aligned}
& d / d t=\partial / \partial t+\boldsymbol{v}_{\perp} \cdot \nabla, \\
& j=-\nabla_{\perp}^{2} \psi-2 n B_{0_{\iota}} / m R
\end{aligned}
$$

is the plasma current density along the $\boldsymbol{e}_{t}$ direction,

$$
U=-\nabla_{\perp}^{2} \phi
$$

is the plasma vorticity, $\mu$ the ion viscosity, $\chi$ the heat conductivity, and $D$ the particle diffusivity. $P=P_{e}=n_{e} T_{e}$, and the subscripts II and $\perp$ denote the parallel and the perpendicular components, respectively. $S_{n}$ and $S_{p}$ are the particle and heat source. $E_{0}$ is the equilibrium electric field for maintaining the original equilibrium plasma current density. The parameters in Equation (1)-(5) are given by $d_{l}=\omega_{c d} / v_{e i}, \Omega=\beta_{e} d_{l}, C_{s}=\left[T_{e} / m_{i}\right]^{1 / 2} /\left(a / \tau_{R}\right)$, and $S=\tau_{R} / \tau_{A}$, where $\beta_{e}=4 \pi n_{e} T_{e} / B_{0 t}^{2}, \omega_{c e}$ is the electron cyclotron frequency, $\mu_{e}$ the electron perpendicular viscosity, $v_{e i}$ the electron-ion collisional frequency, $\tau_{A}=a / V_{A}$ the Alfvén time, and $V_{A}$ is defined using the toroidal field. Cold ions are assumed.

Equations (1)-(8) are the same as those used in Reference [32-34], but the electron inertia and the perpendicular viscosity have been additionally included into Ohm's law, the $3^{\text {rd }}$ and $4^{\text {th }}$ terms on the right hand of equation (2). 


\section{Numerical results}

\subsection{Fast sawtooth crash}

The following simulations are based on typical ASDEX Upgrade experimental parameters, except mentioned elsewhere. The toroidal magnetic field is $B_{0}=2 \mathrm{~T}$, the plasma minor and major radius are $a=0.5 \mathrm{~m}$ and $R=1.7 \mathrm{~m}$, respectively. At the $q=1$ resonant surface the electron temperature is $T_{e}=2 \mathrm{keV}$, and the electron density $n_{e}=3 \times 10^{19} \mathrm{~m}^{-3}$. These parameters lead to $S=2.65 \times 10^{8}, \quad \Omega=9.43 \times 10^{4}, \quad C_{s}=2.05 \times 10^{7}\left(a / \tau_{R}\right), \quad d_{l}=3.12 \times 10^{7}, \quad \chi_{\mid \Gamma}=1.5 \times 10^{10}\left(a^{2} / \tau_{R}\right), \quad$ and $v_{e i}=2.2 \times 10^{4} / s$. Assuming the perpendicular electron thermal heat diffusivity and the ion viscosity to be at an anomalous transport level, e.g., $0.2 \mathrm{~m}^{2} / \mathrm{s}$, one finds $\chi_{\perp}=\mu=18.8\left(\mathrm{a}^{2} / \tau_{R}\right)$ in normalized units. Furthermore, $\mu_{e} / v_{e i}=10^{-4} a^{2}$ and $D_{\perp}=\mu / 5$ are taken. Only a single helicity, $m / n=1 / 1$, is considered. A monotonic profile for the safety factor $q$ is assumed with $q_{0}=0.91$ and $r_{q=1}=0.3 a$, where $q_{0}$ is the safety factor at the magnetic axis, and $r_{q=1}$ the minor radius of the $\mathrm{q}=1$ surface. The initial equilibrium electron density is assumed to be constant along the minor radius, and the equilibrium electron temperature profile is assumed to be

$$
T_{e}=T_{e 0}\left[1-(r / a)^{2}\right]^{\kappa}
$$

where $\kappa$ is an input parameter to define the original equilibrium electron temperature profile.

Figure 1 shows the contours of constant helical flux, $\psi$, at different times, $t=8760$ (a), 8851 (b), 8956 (c), and $9294 \tau_{A}$ (d) for $\kappa=2$. A growing $\mathrm{m} / \mathrm{n}=1 / 1$ magnetic island is seen, accompanied by a shrinking of the original core. The plasma core becomes elliptic later during the nonlinear phase. At $t=9294 \tau_{A}(\mathrm{~d})$, the original core disappears.

At $t=8851 \tau_{A}$ corresponding to figure $1(\mathrm{~b})$, the contours of constant electron temperature and stream function $\phi$ are shown in figure 2 (a) and (b). The electron temperature is normalized to the original electron temperature at $r=0$, and the stream function is normalized to $a^{2} / \tau_{R}$. At a 
later time, $t=8956 \tau_{A}$, corresponding to figure 1 (c), the contours of constant electron temperature and stream function are shown in figure 2 (c) and (d). The electron temperature contours are similar to that of the helical flux shown in figure 1, indicating the fast parallel heat transport, but the radial gradient of the electron temperature near the island's $\mathrm{x}$-point significantly increases, reaching a value more than 10 times larger compared to the original one. Strong vortex flow is built up inside the $q=1$ surface.

The time evolution of the helical flux at the $\mathrm{x}$ - and the o-point of the island as well as at the original magnetic axis are shown in figure 3. The helical flux at the island's o-point and at the axis change little, as expected. The helical flux at the x-point varies from that at the island's opoint to that at the axis, indicating full magnetic reconnection. The reconnection process takes a time period less than $100 \mu \mathrm{s}\left(1 \tau_{A}=8.7 \times 10^{-8} \mathrm{~s}\right)$ when neglecting the small flux change in the early phase when the island is still small. The corresponding time evolution of the radial island width and the radial displacement of the original magnetic axis are shown in figure 4 . The island width is about two times the radial core displacement, but it is larger than two times of the minor radius of the original $q=1$ surface before the core disappears. The change of the central plasma current density profile during sawtooth crash causes a slightly outward shift of the $q=1$ surface as shown in figure 6 .

Figure 5 presents the radial profiles of the $m / n=0 / 0$ component of the electron temperature at different times, showing the flattening of the electron temperature in the central region in a short time period, in agreement with ASDEX Upgrade experimental results [19]. The electron temperature profile becomes hollow at $t=9133 \tau_{A}$ due to the radial hot core displacement towards the $\mathrm{q}=1$ surface, as seen from figure $2(\mathrm{c})$. The local radial profiles of $q_{0 / 0}$, the averaged safety factor calculated by using only the $m / n=0 / 0$ component poloidal field, are shown in figure 6 at different times. The flattening of the plasma current density in the central region leads to the outwards shift of the $q_{0 / 0}=1$ surface. It should be mentioned that $q_{0 / 0}$ is of course 
not the real safety factor in the island region.

Similar to the single fluid case [35], secondary islands are often seen as well in two-fluid simulations for sufficiently high values of $S$ and low values of the perpendicular electron viscosity $\mu_{e}$. The formation of the secondary islands are due to the thin current layer formed during the nonlinear phase, which becomes tearing unstable and breaks up [31,35-39]. However, secondary islands do not significantly affect the sawtooth crash time.

The amplitudes of high harmonic perturbations are usually found to be comparable to that of the $1 / 1$ component during the crash, as seen in experiments [19,20]. Fig. 7 shows the radial profiles of the normalized (to $a B_{0 t}$ ) flux functions $\psi_{1 / 1}, \psi_{2 / 2}, \psi_{3 / 3}$ and $\psi_{4 / 4}$ for the simulation of figure 3 at the time $t=8919 \tau_{A}$. The solid (dashed) curves represent the real (imaginary) part. The amplitude of the $2 / 2$ component is about half of that of the $1 / 1$ component.

\subsection{Sheared plasma rotation and parallel electric field induced by sawtooth crashes}

The internal kink mode always generated an $m / n=0 / 0$ sheared plasma rotation in our simulations. This can be explained by the difference between the mode frequency and the electron fluid frequency, as shown in figure 8 , where the linear mode growth rate and mode frequency are shown as a function of the local equilibrium electron diamagnetic drift frequency $\omega_{* e 0}$ (normalized to $\tau_{R}$ ) at the $q=1$ surface, obtained for $C_{s}=\mu_{e}=0$ and zero electron inertia. The mode becomes stable for a sufficiently large value of $\omega *_{e} 0$, indicating the stabilizing effect of the electron diamagnetic drift. The radial profiles of the radial ion velocity perturbation in the linear phase is shown in figure 9 for $\omega_{*}=5.0 \times 10^{4} / \tau_{\mathrm{R}}\left(\Omega=10^{4}\right.$, linearly unstable $)$ and $7.0 \times 10^{5} / \tau_{\mathrm{R}}\left(\Omega=1.4 \times 10^{5}\right.$, linearly stable $)$. The mode structure is similar to that of the resistive internal kink mode for a small $\omega_{*_{e} 0}$ but to that of the drift-tearing mode for a sufficiently large $\omega *_{e 0}$ [32]. The singular layer width is larger for a larger $\omega_{*_{e} 0}$. It is also seen from figure 8 that 
the mode frequency does not linearly increase with $\omega_{*_{e} 0}$. The difference between the mode frequency and $\omega_{* e}$ leads to a plasma current density perturbation being out of phase with $\psi$. It thus results in an $m / n=0 / 0$ component of an electromagnetic torque $r \cdot \boldsymbol{j} \times \boldsymbol{B}$, localized around the $q=1$ surface and driving a sheared rotation. Such a mechanism is similar to the case of the effect of resonant magnetic perturbations on tearing modes [40]. The radial profile of the $m / n=0 / 0$ component of the poloidal electromagnetic torque in the linear phase is shown in figure 10 for $\omega_{*_{e} 0}=5.0 \times 10^{4} \tau_{R}\left(\Omega=10^{4}\right)$. When integrating over the plasma volume, both the total torque and the total plasma angular momentum vanish, since there is no momentum source in these calculations.

In the nonlinear phase, both the q-profile and the electron pressure profile significantly change, leading to a change of the electromagnetic force and thus of the sheared plasma rotation. The radial profiles of the $m / n=0 / 0$ component of poloidal plasma rotation velocity at different times are shown in figure 11. The driven plasma rotation is in the counter (co-) current direction inside (outside) the $q=1$ surface in the linear phase, and propagates towards the magnetic axis during the nonlinear phase. After the sawtooth crash, the driven plasma rotation is in the co- (counter-) current direction inside (outside) the $q=1$ surface, in agreement with TCV experimental observations [41,42] (The equilibrium plasma current density is in negative direction in our calculations). The amplitude of the rotation velocity reaches the level of the electron diamagnetic drift velocity.

During the sawtooth crash, strong parallel electric fields are induced at the x-point. Contours of the negative parallel electric field, $-E_{\|}=d \psi / d t$ (in the unit $a B_{0 t} / \tau_{R}$ ), are shown at different times, $t=8760$ (a), 8813 (b), 8851 (c), and $8956 \tau_{A}(\mathrm{~d})$ in figure 12 . The original equilibrium electric field is $-0.55\left(a B_{0} / \tau_{R}\right)$. The parallel electric field is increased during the sawtooth crash compared to the equilibrium field by about 1000 times in the island's X-point region. The maximum electron velocity produced by the reconnection can be computed by 
neglecting collisions and relativistic effects. From the equation of motion for a single electron,

$$
m_{e} \frac{d}{d t} v_{f}=-e E_{||}=e \frac{d}{d t} \psi
$$

one finds by integration that

$$
v_{f}=\frac{e}{m_{e}}(\Delta \psi)_{r e c}
$$

if neglecting the initial electron speed ( $v_{f}$ is the parallel electron speed, $e$ is the electron electric charge, and $m_{e}$ is the electron mass). Using the reconnected flux $(\Delta \psi)_{\text {rec }}=0.0006\left(a B_{t}\right)$ from figure 3 and the input parameters mentioned before, $a=0.5 \mathrm{~m}$ and $B_{0 t}=2.0 \mathrm{~T}$, an electron velocity of $v_{f}=1.05 \times 10^{8} \mathrm{~m} / \mathrm{s}$ is found, corresponding to an electron energy of $31.6 \mathrm{keV}$ and a collision time of $1.3 \mathrm{~ms}$. This indicates that supra-thermal electrons can be generated during the sawtooth crash in existing tokamak plasmas as observed in experiments $[43,44]$. It should be kept in mind, however, that the above estimation only gives an upper limit, and a kinetic treatment is required to accurately calculate the induced super-thermal electron energy and distribution in such a process.

For a full reconnection the reconnected flux is approximately given by [6]

$$
(\Delta \psi)_{r e c} \approx\left(1-q_{0}\right) B_{p s} r_{q=1} / 4=\left(1-q_{0}\right) B_{0 t}\left(r_{q=1}\right)^{2} /(4 R),
$$

where $B_{p s}$ is the poloidal field at the $q=1$ surface. A $q$-profile with a smaller value of the safety factor at the magnetic axis, $q_{0}=0.71$, (but keeping $r_{q=1}=0.3 a$ ) would result in $(\Delta \psi)_{\text {rec }}=0.0026$ $\left(a B_{t}\right)$ and an order of magnitude increased electron energy. For such a case, contours of the helical flux (a and c) and of the (negative) parallel electric field (b and d) are shown at two different times in figure 13.

As the generated fast electron energy is proportional to the square of $(\Delta \psi)_{\text {rec }}$ and thus scales with $\left(a B_{0 t}\right)^{2}$, relativistic runaway electrons could readily be generated during sawtooth crashes in ITER or a fusion reactor, serving as seed for an avalanche in case of a subsequent disruption. 


\subsection{Effect of plasma parameters on sawtooth crash}

Parameters scans have been carried out in order to investigate their influence on the sawtooth crash time. For sufficiently high $\mathrm{S}$ values the crash time is no longer affected by changes in $S$ as expected for collisionless reconnection. Decreasing the value of $S$ by one order of magnitude to $S=2.65 \times 10^{7}$ essentially showed the same results. For vanishing electron viscosity but finite electron inertia, the calculated sawtooth crash time and the plasma current layer width are very similar to the case for $\mu_{e} / v_{e i}=10^{-4} a^{2}$. Electron viscosity matters if electron inertia is neglected. In that case, the crash time increases for vanishing electron viscosity, and plasmoids appear for low electron viscosity. These findings suggest that the electron inertia and the parallel electron pressure gradient terms in the generalized Ohm's law are the most important terms in determining the sawtooth crash time. Furthermore, a simple estimation demonstrates that the electron inertia term dominates over the electron viscosity in the nonlinear phase: Using $j^{\prime} \sim j / \delta, j^{\prime \prime}=j / \delta^{2}$ with the radial current layer width $\delta=0.0025 a$ and the radial velocity $v_{r}\left(=1.5 \times 10^{5} \mathrm{a} / \tau_{R}\right)$ obtained from our numerical results, and $\mu_{e} / v_{e i}=10^{-4} a^{2}$, the amplitude of the electron inertia term in the generalized Ohm's law is estimated to be about 10 times larger than the viscosity term in the nonlinear phase. The effects of the parallel electron pressure gradient can be divided into two contributions: one is the so-called finite $\rho_{s}$ effect, and the other is the electron diamagnetic drift due to the radial electron pressure gradient, where $\rho_{s}$ is the ion Larmor radius defined with the electron temperature. The island growth is found to be faster for larger values of $\rho_{s}$, while a large electron diamagnetic drift is stabilizing, as shown in figure 8 and in Ref. [31].

Using the same q-profile as before, but a steeper temperature profile (larger $\kappa$ value and thus larger electron diamagnetic drift), the time evolution of the electron temperature at the minor radius both inside and outside the $q=1$ surface is shown in figure 14 for $\kappa=3$ and 8 . 
When the island width is still small, the island growth is much faster for a smaller value of $\kappa$ (or $\omega *_{e 0}$ ) due to a weaker stabilizing effect of the diamagnetic drift. However, a fast decrease in the electron temperature is found for both cases once the island width is sufficiently large. This can be explained by the flattening of the electron temperature profile inside a large island due to the fast parallel heat transport, such that the stabilizing effect of the electron diamagnetic drift decreases with increasing island width.

Using a different q-profile with a larger $q_{0}\left(q_{0}=0.95\right)$ and $r_{q=1}=0.21 a$, the time evolution of the electron temperature both inside and outside the $q=1$ surface is shown in figure 15 for $T_{e}=300,500$ and $2000 \mathrm{eV}$. The mode growth is much faster in the linear phase for a lower $T_{e}$. Once the electron temperature begins to change, however, it decreases faster for $T_{e}=2 \mathrm{keV}$ than in case of $T_{e}=300$ and $500 \mathrm{eV}$, indicating that the finite $\rho_{s}$ effect dominates the sawtooth crash time for $T_{e}=2000 \mathrm{eV}\left(\rho_{s}=0.0092 a\right)$.

Slow sawtooth crashes are found only for a sufficiently small $\left(1-q_{0}\right)$ and a large local electron diamagnetic drift frequency at the $q=1$ surface. Figure 16 shows the time evolution of the electron temperature both inside and outside the $q=1$ surface for $q_{0}=0.95$ and $r_{q=1}=0.21 a$. In this case, a much longer crash time, $\sim 10^{4} \tau_{A} \sim 10^{-3} \mathrm{~s}$, is found for $\kappa=4$ and 6. In the hybrid operational mode with small $\left(1-q_{0}\right)$ and a weak magnetic shear in the central region, slow sawtooth crashes [45] or even continuous $1 / 1$ mode activity [46] have been observed in experiments. The dependence on $\left(1-q_{0}\right)$ indicates the role of the local magnetic shear around the $\mathrm{q}=1$ surface as found in analytic theory [e.g. 14]. For small values of $\left(1-q_{0}\right)$, it approximately equals the local shear $\mathrm{rq}^{\prime} / \mathrm{q}$ at the $\mathrm{q}=1$ surface. Comparing the results shown in figures 14 and 16 , the critical island width, above which the electron temperature rapidly decreases, is found to be larger for a smaller magnetic shear. This is consistent with the fact that the parallel heat transport is slower for smaller island width and magnetic shear [e.g. 47, 48], such that the diamagnetic drift has a stabilizing effect up to a larger island width. 


\section{Discussion and summary}

Using the large aspect ratio approximation and realistic input parameters for ASDEXUpgrade, extensive calculations have been carried out in order to investigate the sawtooth crash time as well as the physics involved. Our physics model includes all the relevant two-fluid effects, such as the electron pressure gradient, electron inertia and perpendicular viscosity in Ohm's law as well as the parallel and perpendicular electron particle and energy transport. We were able to reproduce important experimental observations, such as the fast sawtooth crash and the resulting mean shear flow. However, we have not found partial reconnection yet. In Ref. [25] it was reported that partial reconnection might occur for a sufficiently large electron pressure and diamagnetic drift frequency, but it was mentioned that the input parameters utilized there are not realistic for tokamak plasmas [25].

The large parallel electric fields associated with the fast reconnection events might be sufficient to generate supra-thermal electrons in medium size tokamaks or even runaway electrons in ITER. It should be noticed, however, that the possible kinetic effect for such super-thermal electrons have not been included in our calculations.

In summary, the growth of $m / n=1 / 1$ magnetic islands is studied using two-fluid equations. It is found that:

(1) For typical ASDEX Upgrade parameters, the sawtooth crash time is about $100 \mu$ s or shorter, in agreement with experimental observations and earlier numerical results [31]. The amplitudes of higher harmonics are quite large, in particular the $m / n=2 / 2$ component helical flux becomes about half of that of the $1 / 1$ component during the crash.

(2) Slow sawtooth crashes were found only for a sufficiently small value of $\left(1-q_{0}\right)$ and large local electron diamagnetic drift frequencies.

(3) Sheared plasma rotation is driven by the internal kink mode, propagating from the original 
$q=1$ surface towards the magnetic axis during the nonlinear phase. After the sawtooth crash, the driven plasma rotation is in the co- (counter-) current direction inside (outside) the $q=1$ surface, in agreement with experiments.

(4) The parallel electric field is increased by three to four orders of magnitude in the island's xpoint region during the sawtooth crash, possibly generating super-thermal electrons during the crash in existing tokamaks and runaway electrons in a fusion reactor. 


\section{References}

[1] S. Von Goeler et al., Phys. Rev. Lett. 33 (1974) 1201.

[2] B. B. Kadomtsev, Sov. J. Plasma Phys. 1 (1975) 389.

[3] A. W. Edwards et al., Phys. Rev. Lett. 57 (1986) 210.

[4] L. McGuire, TFTR Group, Phys. Fluids B 2 (1990) 1287.

[5] J. A. Wesson et al., Nucl. Fusion 31 (1990) 111.

[6] J. A. Wesson, Nucl. Fusion 30 (1990) 2545.

[7] J. F. Drake and R. G. Kleva, Phys. Rev. Lett. 66 (1991) 1458.

[8] M. Ottaviani and F. Porcelli, Phys. Rev. Lett. 71 (1993) 3802.

[9] D. Biskamp and J. F. Drake, Phys. Rev. Lett. 73 (1994) 971.

[10] J. F. Drake et al., Phys. Rev. Lett. 73 (1994) 1251.

[11] F. Waelbroeck, Phys. Fluids B 1 (1989) 2372.

[12] L. E. Zakharov et al., Phys. Fluids B 5 (1993) 2498.

[13] D. Biskamp, Phys. Fluids B 3 (1991) 3353.

[14] X. Wang and A. Bhattarchajee, Phys. Rev. Lett. 70 (1993) 1627.

[15] F. Porcelli, Phys. Rev. Lett. 66 (1991) 425.

[16] A. Y. Aydemir, Phys. Fluids B 4 (1992) 3469.

[17] Qingquan Yu, Nucl. Fusion 35 (1995) 1012.

[18] A. Skyes and J. A. Wesson, Phys. Rev. Lett. 37 (1976) 140.

[19] V. Igochine, J. Boom, I. Classen et al., Phys. Plasmas 17, 122506 (2010).

[20] X. Xu, J. Wang, Y. Wen et al., Plasma Phys. Control. Fusion, 52, 015008 (2010).

[21] F. Porcelli, D. Boucher and M. N. Rosenbluth, Plasma Phys. Control. Fusion, 38, 2163 (1996).

[22] F. D. Halpern, D. Leblond, H. Lütjens and J-F Luciani, Plasma Phys. Control. Fusion, 53, 015001 (2011).

[23] F. D. Halpern, H. Lütjens and J-F Luciani, Phys. Plasmas 18, 102501 (2011).

[24] D. Biskamp, Phys. Fluids 29, 1520 (1986)

[25] D. Biskamp and T. Sato, Phys. Plasmas 4, 1326 (1997))

[26] M. N. Rosenbluth, R. Y. Dagazian, and P. H. Ruthford, Phys. fluids 16, 1894 (1973)

[27] R. J. Buttery, T. C. Hender, D. F. Howell, R. J. La Hay et al., Nucl. Fusion 43, 69 (2003)

[28] V. Igochine, A. Gude, S. Günter, K. Lackner, Q. Yu, et al., Phys. Plasmas 21, 110702 (2014). 
[29] P. A. Sweet, in Electromagnetic Phenomena in Cosmic Physics (B. Lehnert Ed.), Cambridge University Press, Cambridge (1958) 123.

[30] E. N. Parker, J. Geophys. Res. 62 (1957) 509.

[31] S. Günter, Q. Yu, K. Lackner, A. Bhattacharjee Y-M Huang, Plasma Phys. Control. Fusion, 57, 014017 (2015).

[32] Q. Yu, Nucl. Fusion 50, 025014 (2010).

[33] Q. Yu and S. Günter, Nucl. Fusion 49, 062001 (2009).

[34] Q. Yu, S. Günter, Y. Kikuchi, and K. H. Finken, Nucl. Fusion 48, 024007 (2008).

[35] Q. Yu, S. Günter and K. Lackner, Nucl. Fusion 48, 024007 (2014).

[36] Yi-Min Huang, A. Bhattacharjee, and Brian P. Sullivan, Phys. Plasmas 18, 072109 (2011)

[37] N. F. Loureiro, A. A. Schekochihin and S. C. Cowley, Phys. Plasmas 14, 100703 (2007)

[38] H. P. Furth, J. Killeen, and M. N. Rosenbluth, Phys. Fluids 6, 456 (1963).

[39] S. Schmidt, S. Günter and K. Lackner, Phys. Plasmas 16, 072302 (2009)

[40] Q. Yu, S. Günter and K.H. Finken, Phys. Plasmas 16, 042301 (2009).

[41] B. P. Duval, A. Bortolom, A. Karpushov, et al., Phys. Plasmas 15, 056113 (2008)

[42] B. P. Duval, A. Bortolom, L. Federspiel, et al., 2010 Proc. 23rd Int. Conf. on Fusion Energy (Daejeon, 2010). http://www-naweb.iaea.org/napc/physics/fec/fec2010/htm1/node316.htm\#66675

[43] P. V. Savrukhin, Phys. Rev. Lett. 86 (2001) 3036.

[44] I. Klimanov, A. Fasoli, T. P. Goodman, and TCV team, Plasma Phys. Control. Fusion, 49, L1-L6 (2007).

[45] P. Buratti, B. Alper, S.V. Annibaldi, et al., Plasma Phys. Control. Fusion, 48, 1005 (2006).

[46] J. Stober, A.C.C. Sips, C. Angioni, et al., Nucl. Fusion 47, 728 (2007).

[47] R. Fitzpatrick, Phys. Plasmas 2, 825 (1995)

[48] Q. Yu, Phys. Plasmas 13, 062310 (2006). 


\section{CAPTION}

Figure 1 Constant- $\psi$ contours in the $R-Z$ plane at $t=8760(\mathrm{a}), 8851(\mathrm{~b}), 8956(\mathrm{c})$, and $9294 \tau_{A}$ (d) for $\kappa=2$, where $R=0$ corresponds to the major radius of the original magnetic axis, and $Z$ is pointing along the vertical direction, being normalized to the plasma minor radius $a$. At $t=9294 \tau_{A}(\mathrm{~d})$, the original core disappears.

Figure 2 For the time $t=8851 \tau_{A}$ of figure $1(\mathrm{~b})$, the contours of constant electron temperature and stream function are shown in (a) and (b). At $t=8956 \tau_{A}$ of figure 1 (c), the contours of constant electron temperature and stream function are shown in (c) and (d). The electron temperature contours are similar to that of the helical flux, indicating the fast parallel heat transport. Strong vortex flow is built up inside the $q=1$ surface.

Figure 3 Time evolution of the normalized (to $a B_{0 t}$ ) helical flux at the $\mathrm{x}$ - and o-points of the island and at the original magnetic axis. The helical flux at the island's o-point and at the original magnetic axis changes little, while the helical flux at the x-point varies from that at the island's o-point to that at the original magnetic axis, indicating a full magnetic reconnection, which takes a time period of less than $100 \mu \mathrm{s}\left(1 \tau_{A}=8.7 \times 10^{-8} \mathrm{~s}\right)$.

Figure 4 Time evolution of the radial island width and the radial displacement of the original magnetic axis for the same simulation as shown in Fig. 3. The island width is about two times of the radial core displacement, but it is larger than two time of the minor radius of the original $q=1$ surface at the end of sawtooth crash.

Figure 5 Radial profiles of the $m / n=0 / 0$ component of the electron temperature at various times for the simulation of Fig. 3. The profiles flatten during a short time period.

Figure 6 Local radial profiles of $q_{0 / 0}$ at various times (corresponding to simulations of Fig. 3 ), where $q_{0 / 0}$ is the averaged safety factor calculated by using only the $m / n=0 / 0$ component poloidal field. The flattening of the plasma current density profile in the central region leads to 
the outwards shift of the $q_{0 / 0}=1$ surface in the nonlinear phase.

Figure 7 Local radial profiles of the normalized (to $a B_{0 t}$ ) perturbed fluxes $\psi_{1 / 1}, \psi_{2 / 2}, \psi_{3 / 3}$ and $\psi_{4 / 4}$ for $t=8919 \tau_{A}$ of Fig. 3. The solid (dashed) curves correspond to the real (imaginary) part.

Figure 8 Linear mode growth rate and mode frequency as a function of the normalized local electron diamagnetic drift frequency $\omega_{*_{e} 0} \tau_{R}$ at the $q=1$ surface, obtained for $C_{s}=\mu_{e}=0$ and zero electron inertia. The mode becomes stable for a sufficiently large $\omega *_{e} 0$, indicating the stabilizing effect of the diamagnetic drift. Note that the mode frequency is not linearly proportional to $\omega *_{e} 0$. Figure 9 Radial profiles of the radial ion velocity perturbation during the linear phase for $\omega *_{e 0}=5.0 \times 10^{4} \tau_{R}\left(\Omega=10^{4}\right.$, linear unstable $)$ and $7.0^{*} 10^{5} \tau_{R}\left(\Omega=1.4 \times 10^{5}\right.$, linear stable $)$. The solid (dashed) curves correspond to the real (imaginary) part. The mode structure is similar to that of the resistive internal kink mode for a small $\omega_{*_{e} 0}$ but to that of a drift-tearing mode for a sufficiently large $\omega_{*_{e} 0}$. The singular layer width is larger for a larger value of $\omega_{*_{e} 0 \text {. }}$.

Figure 10 Radial profile of the $m / n=0 / 0$ component of electromagnetic torque in the linear phase for $\omega_{*_{e} 0}=5.0 \times 10^{4} \tau_{R}\left(\Omega=10^{4}\right)$. The total torque, obtained by integrating over the plasma volume, vanishes as no momentum input is provided.

Figure 11 Radial profiles of the $m / n=0 / 0$ component of the poloidal plasma rotation velocity $V_{p}$ for various times in the simulation of figure 3 . The driven plasma rotation is in the counter (co-) current direction inside (outside) the $q=1$ surface in the linear phase and propagates towards the magnetic axis during the nonlinear phase. After the sawtooth crash, the driven plasma rotation is in the co- (counter-) current direction inside (outside) the $q=1$ surface.

Figure 12 Contours of the negative parallel electric field, $-E_{\|}=d \psi / d t$ (in the unit $a B_{0} / \tau_{R}$ ), at $t=8760$ (a), $8813(\mathrm{~b}), 8851$ (c), and $8956 \tau_{A}(\mathrm{~d})$, corresponding to figure 3 . The original equilibrium electric field is $-0.55\left(a B_{0} / \tau_{R}\right)$. The parallel electric field is increased by about 1000 times in the island's x-point region during the sawtooth crash. 
Figure 13 Contours of the helical flux ( $\mathrm{a}$ and $\mathrm{c}$ ) and the negative parallel electric field (b and d) are shown at two different time during the mode growth for $q_{0}=0.71$ and $r_{q=1}=0.3 a$. The original equilibrium electric field is $-0.55\left(a B_{0 t} / \tau_{R}\right)$. The parallel electric field is increased by four orders of magnitude in the island's x-point region during the sawtooth crash.

Figure 14 Time evolution of the $\mathrm{m} / \mathrm{n}=0 / 0$ component electron temperature at minor radius inside and outside the $q=1$ surface for $\kappa=3$ and 8 , using the same q-profile as for figure 3 . When the island width is small, the island growth is much faster for a smaller value of $\kappa$ (or $\left.\omega *_{e}\right)$ ). Fast electron temperature decrease is found however for both cases when the island width is sufficiently large.

Figure 15 Time evolution of the $\mathrm{m} / \mathrm{n}=0 / 0$ component electron temperature at minor radius inside and outside the $q=1$ surface for $T_{e}=300,500$, and $2000 \mathrm{keV}$, obtained with $q_{0}=0.95$ and $r_{q=1}=0.21 \mathrm{a}$. The electron temperature decreases faster for $T_{e}=2 \mathrm{keV}$ than for $T e=300$ and 500 $\mathrm{eV}$, once the electron temperature begins to change.

Figure 16 Time evolution of the $\mathrm{m} / \mathrm{n}=0 / 0$ component electron temperature at minor radius inside and outside the $q=1$ surface for $\kappa=4$ and 6 , obtained with $q_{0}=0.95$ and $r_{q=1}=0.21 a$. In this case the crash time is much longer. 
Figure 1
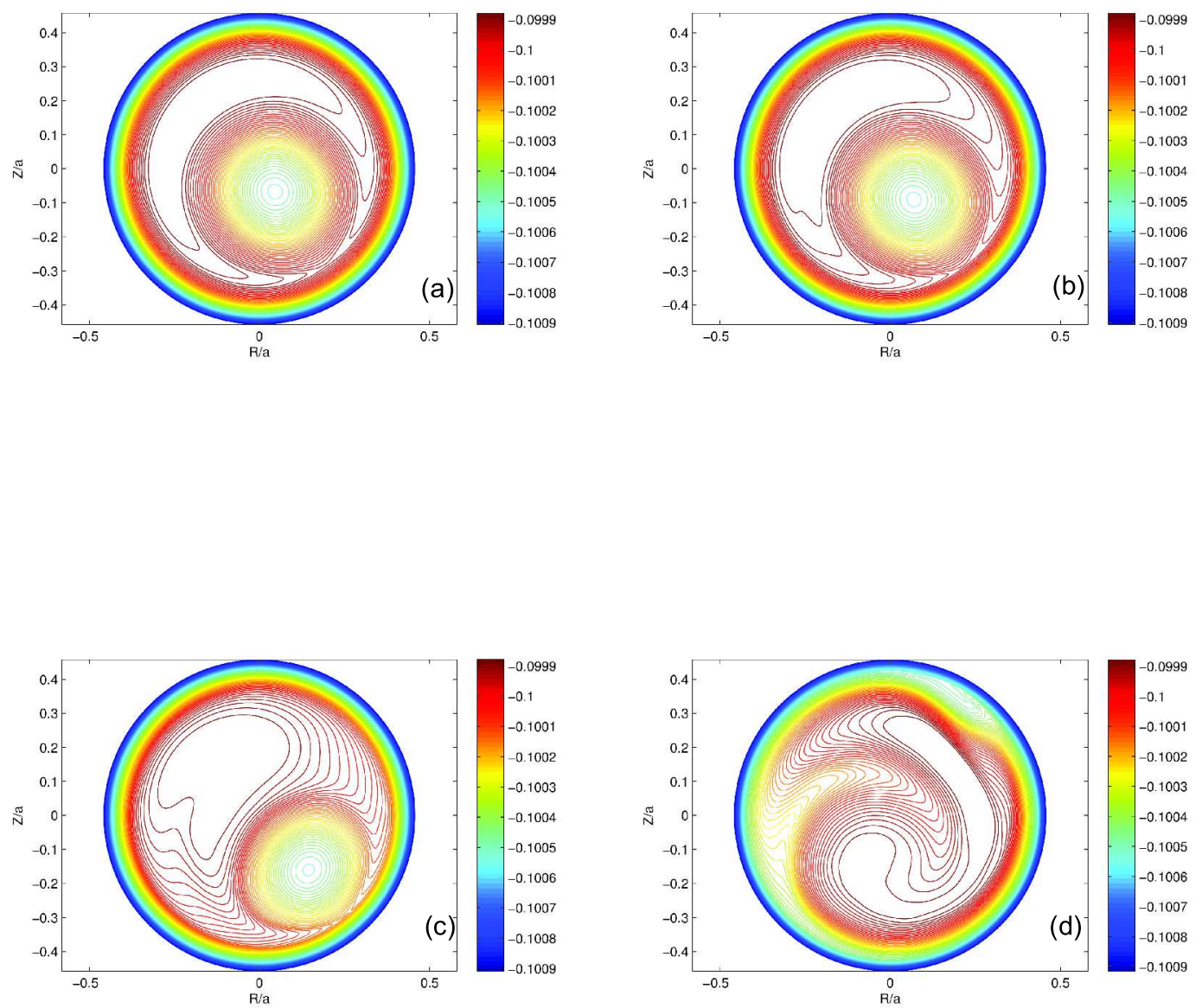
Figure 2
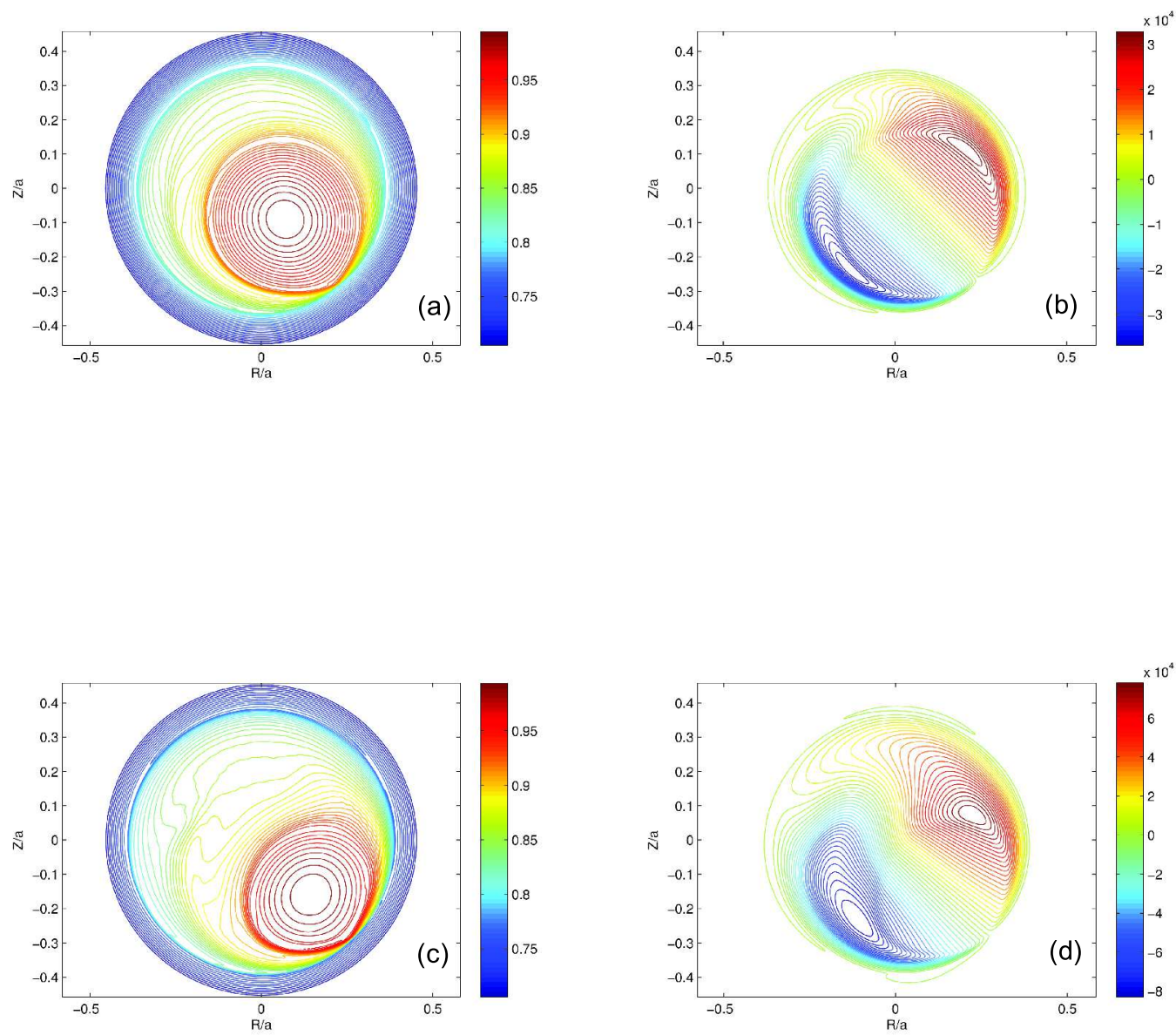


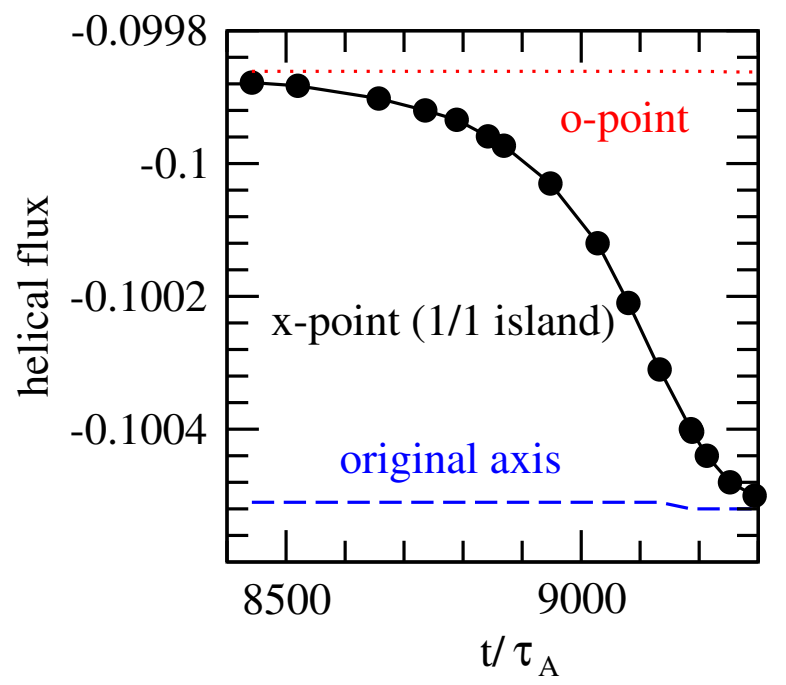

Figure 3 


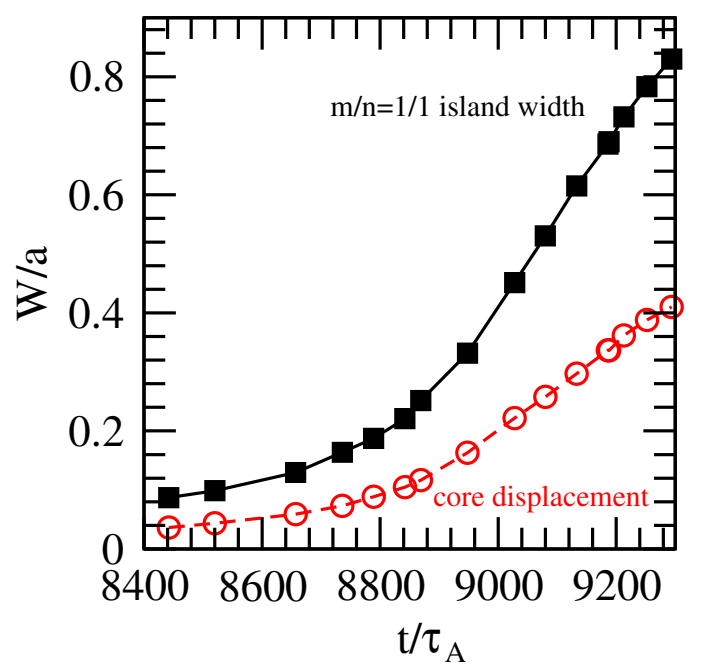

Figure 4 


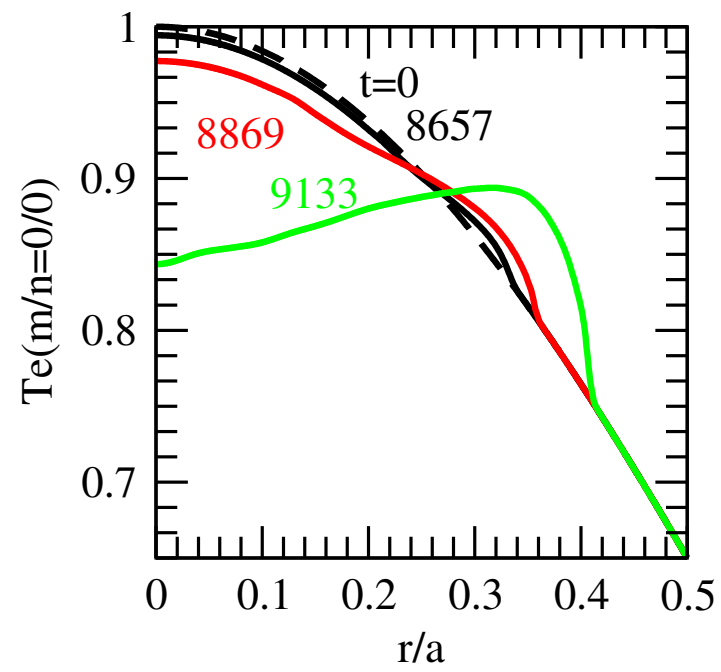

Figure 5 


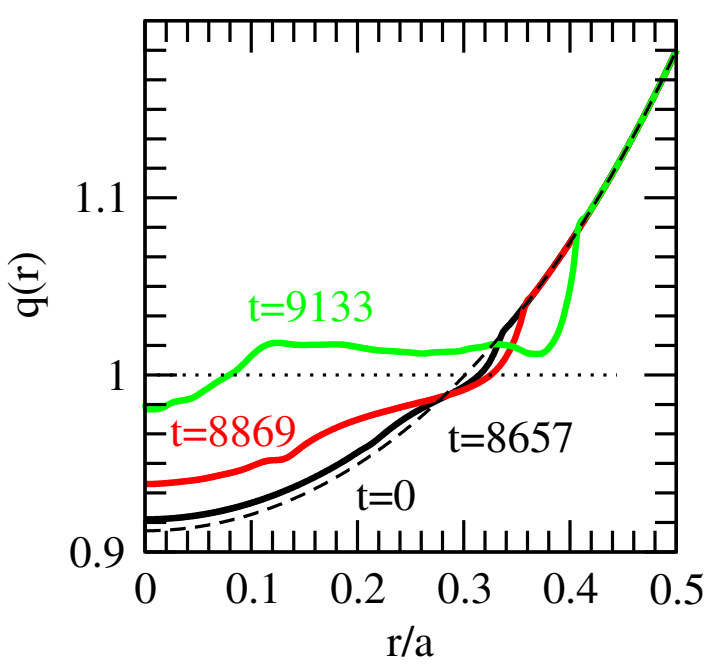

Figure 6 


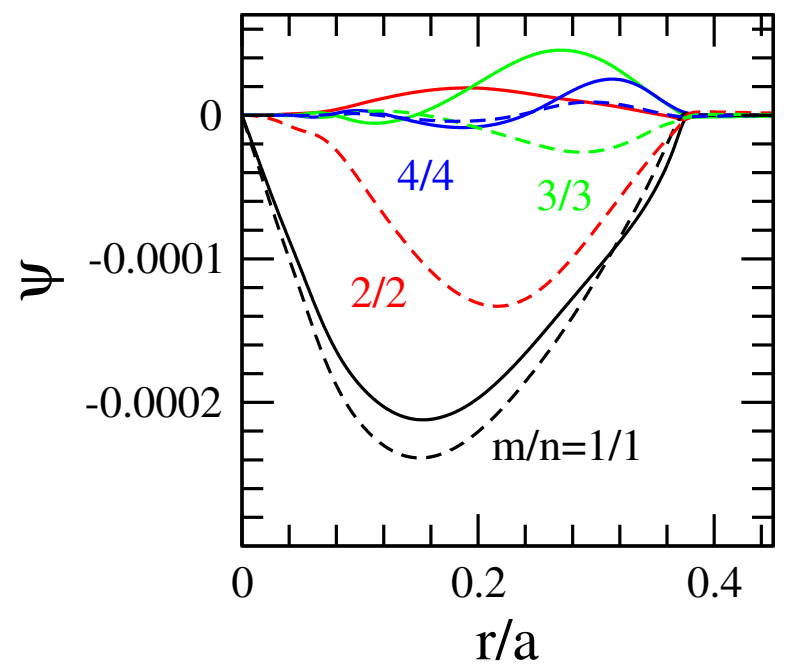

Figure 7 


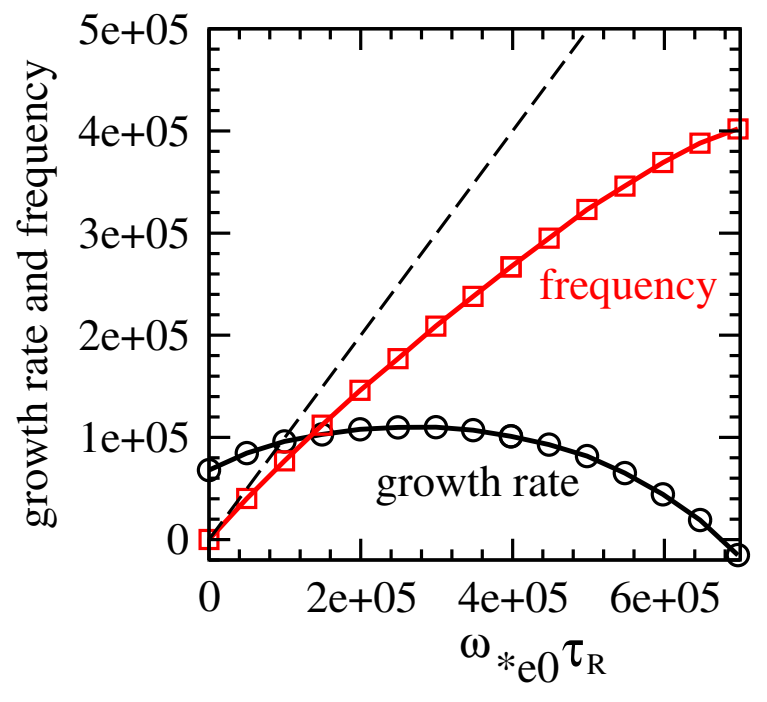

Figure 8 


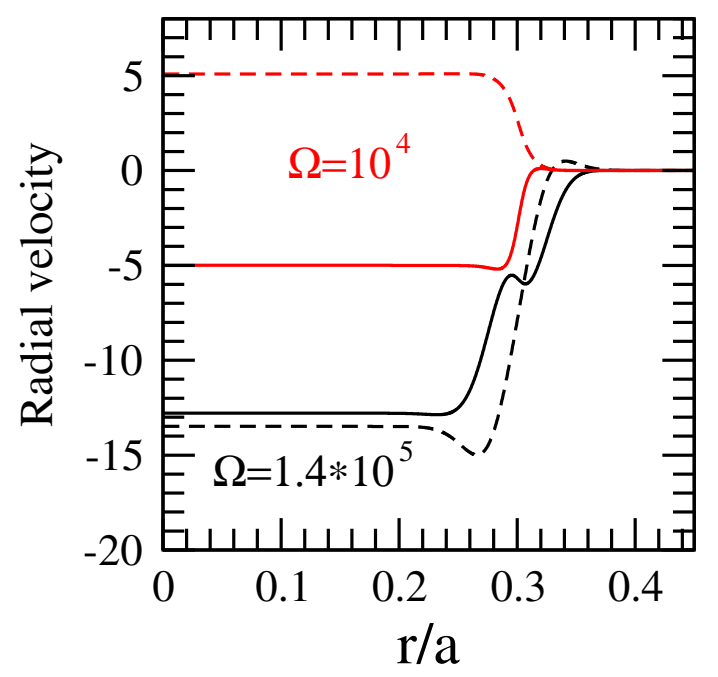

Figure 9 


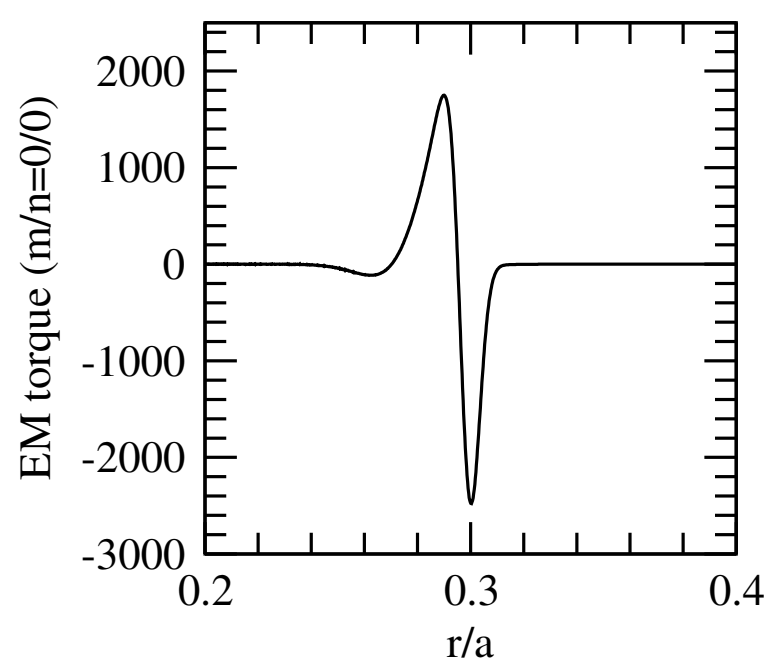

Figure 10 


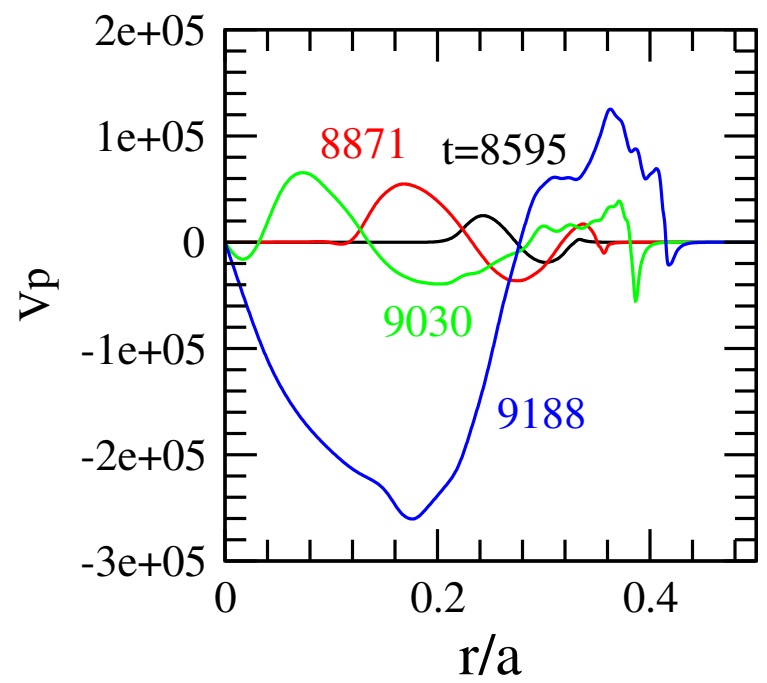

Figure 11 
Figure 12
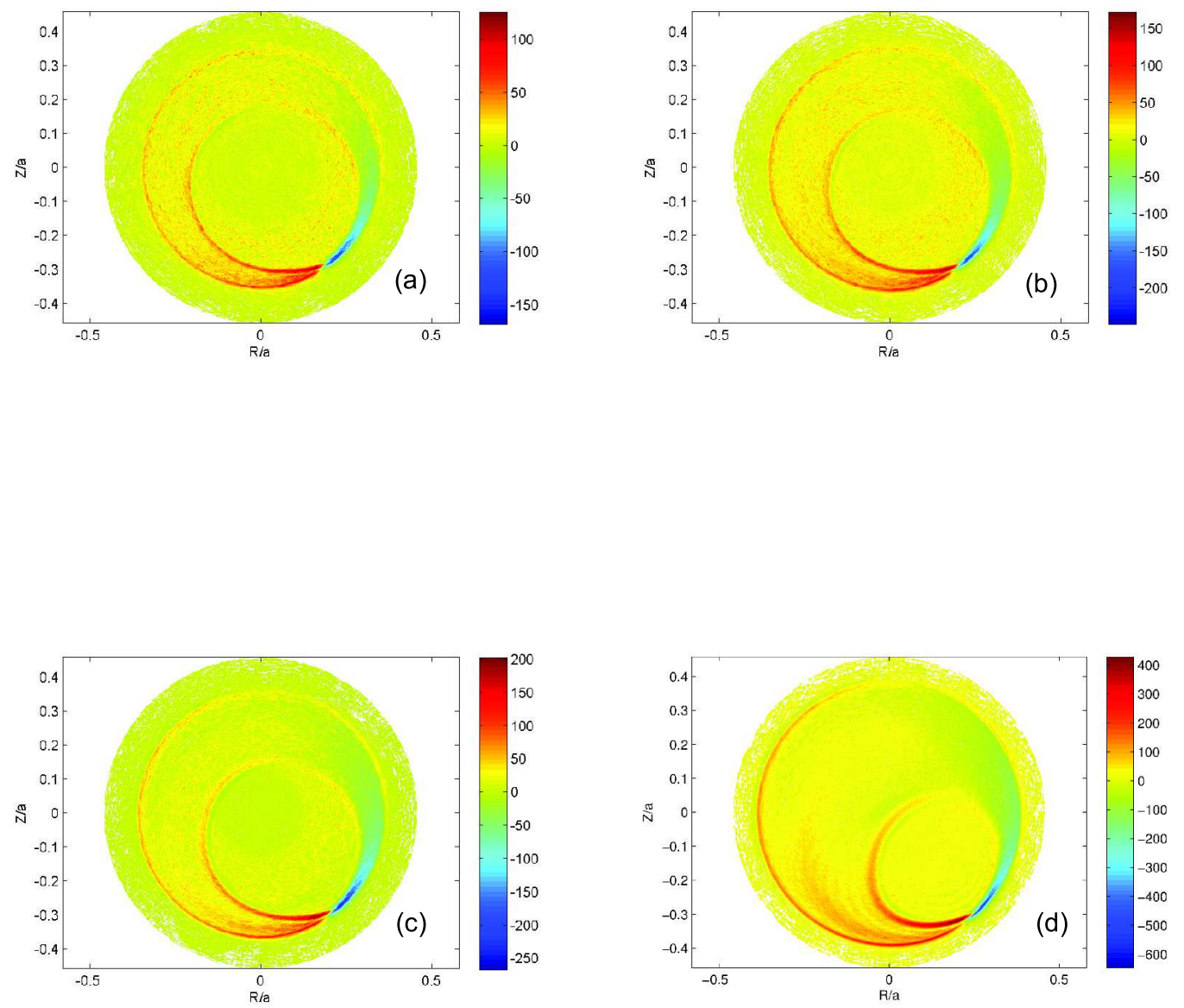
Figure 13
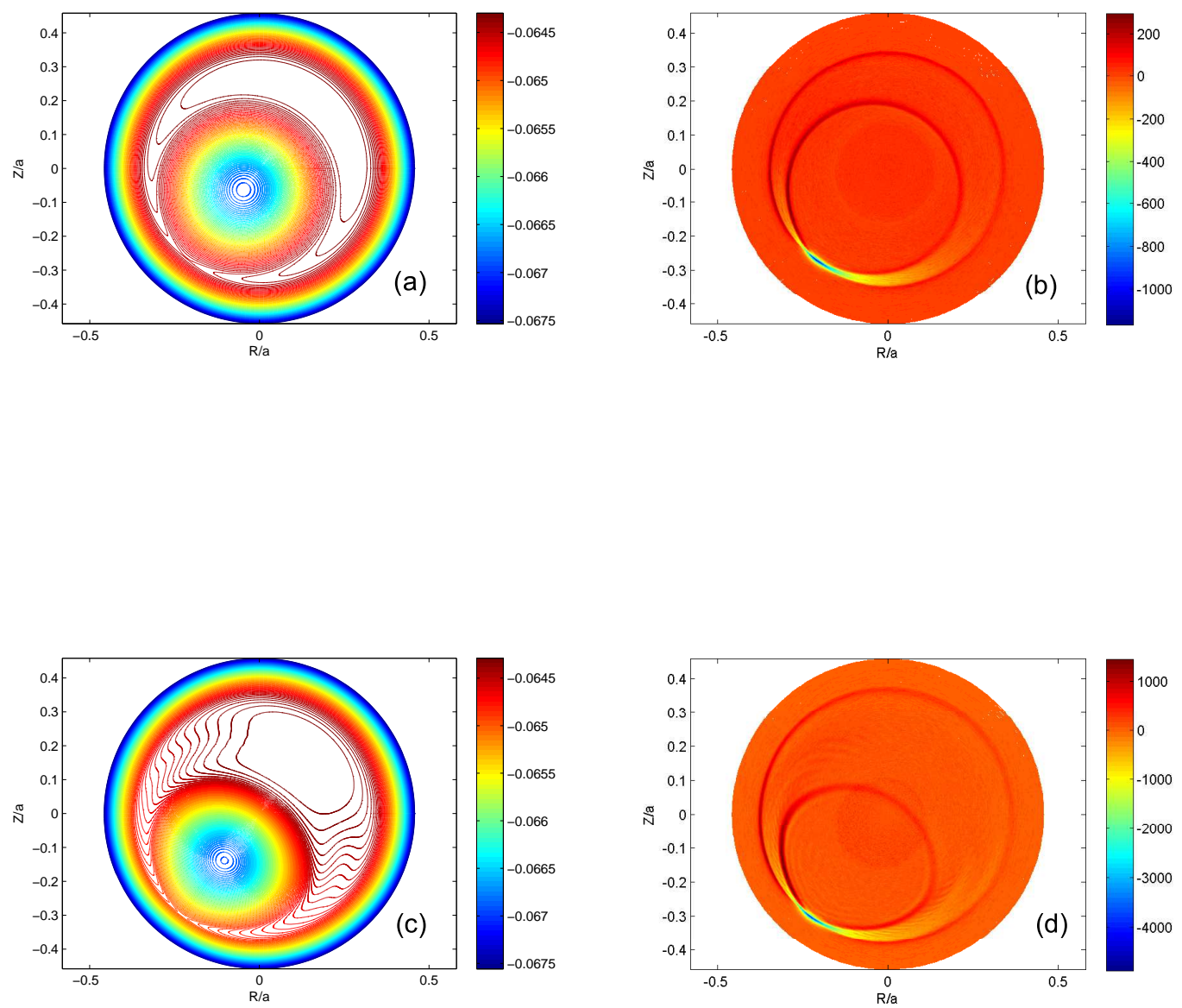


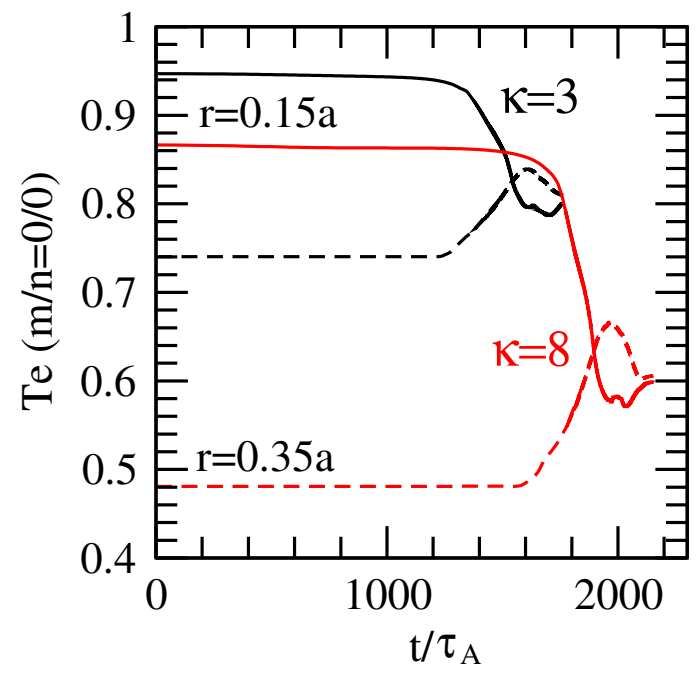

Figure 14 


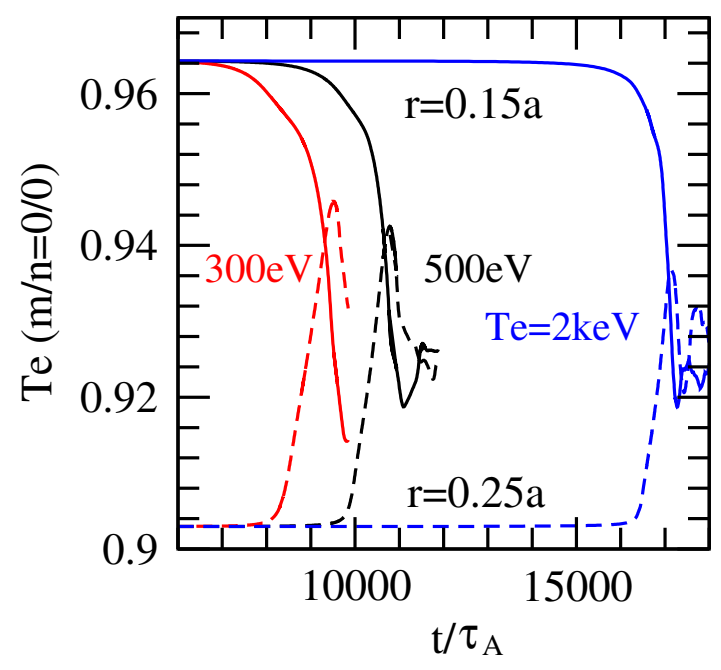

Figure 15 


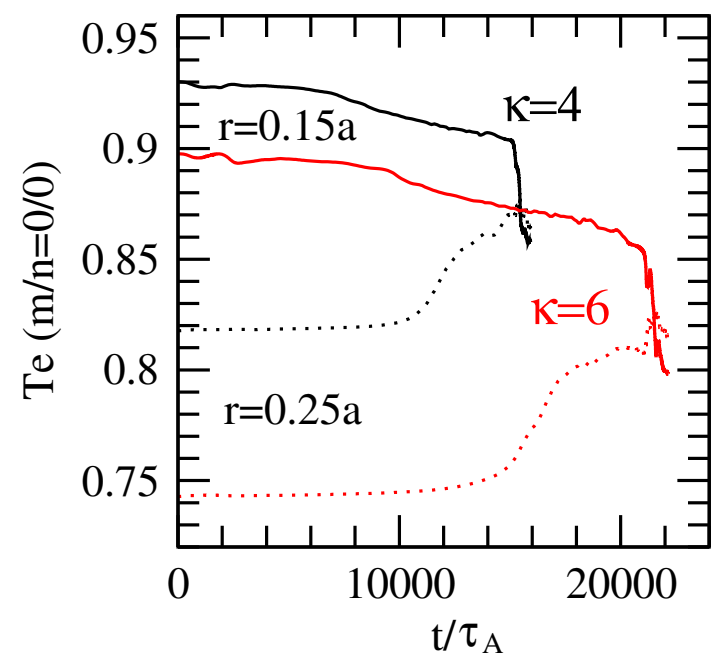

Figure 16 